\title{
Observational constraints for lithium depletion before the RGB ${ }^{\star}$
}

\author{
P. de Laverny ${ }^{1}$, J. D. do Nascimento Jr. ${ }^{2}$, A. Lèbre ${ }^{3}$, and J. R. De Medeiros ${ }^{2}$ \\ 1 Observatoire de la Côte d'Azur, Département Fresnel, UMR 6528 CNRS, BP 4229, 06304 Nice, France \\ 2 Departamento de Física, Universidade Federal do Rio Grande do Norte, 59072-970 Natal, RN., Brazil \\ 3 Groupe d'Astrophysique, UMR 5024/CNRS, U. de Montpellier, Place Bataillon, 34095 Montpellier, France
}

Received 4 February 2003 / Accepted 18 April 2003

\begin{abstract}
Precise Li abundances are determined for 54 giant stars mostly evolving across the Hertzsprung gap. We combine these data with rotational velocity and with information related to the deepening of the convective zone of the stars to analyse their link to Li dilution in the referred spectral region. A sudden decline in Li abundance paralleling the one already established in rotation is quite clear. Following similar results for other stellar luminosity classes and spectral regions, there is no linear relation between $\mathrm{Li}$ abundance and rotation, in spite of the fact that most of the fast rotators present high Li content. The effects of convection in driving the Li dilution is also quite clear. Stars with high Li content are mostly those with an undeveloped convective zone, whereas stars with a developed convective zone present clear sign of Li dilution.
\end{abstract}

Key words. stars: abundances - stars: evolution - stars: interiors - stars: late-type - stars: rotation

\section{Introduction}

Despite the important advances made in the past decade in the study of the stellar lithium behavior, a large number of questions are not yet answered. We do not completely understand the processes controlling lithium production, nor how and when lithium is depleted. Related to these questions are the physical bases of the mixing mechanisms in stellar interiors. Following the initial study by Bonsack (1959) different works have attempted to establish the behavior of lithium abundance along the giant branch (e.g. Alschuler 1975; Wallerstein 1966; Brown et al. 1989) and its link to rotation (Wallerstein et al. 1994; De Medeiros et al. 2000). These works have shown a steady decline in lithium abundances from spectral types F5III to F8III, a wide spread around G0III and a gradual decline with temperature for stars redward of such spectral type. Wallerstein et al. (1994) have found that giant stars with $v \sin i>50 \mathrm{~km} \mathrm{~s}^{-1}$, located in the $(B-V)$ color interval from 0.40 to 0.70 , present lithium abundance close to the presumed primordial value, whereas slower rotators show reduced lithium abundances in spite of their earlier spectral types. De Medeiros et al. (2000) have found a trend of discontinuity in the distribution of the lithium abundances around the spectral type G0III, paralleling the sudden decline observed in rotational velocity. The origin of such a discontinuity is not yet well established but it seems to strongly depend on stellar mass (De Medeiros \& Mayor 1990).

Send offprint requests to: J. R. De Medeiros,

e-mail: renan@dfte.ufrn.br

* Based on observations collected at ESO, La Silla, Chile, and at the Observatoire de Haute Provence, France, operated by the Centre National de la Recherche Scientifique (CNRS).
Nevertheless, the rotation-lithium connection in giant stars appears to be a more complicated problem. In spite of the fact that high lithium content is associated with fast rotation, slow rotators present a large spread in the values of lithium abundance (De Medeiros et al. 2000). In addition, this connection seems to depend on the stellar mass, metallicity and age. Another important question concerns the level of dilution of lithium along the giant branch. While standard theory predicts a factor of dilution of about 40 to 60 for $1 M_{\odot}$ and $2 M_{\odot}$ respectively (Iben 1965a, 1965b), the observations for these stellar masses show that the factor of dilution of lithium is far higher, reaching values as large as 400 to 1000 . A more solid discussion about this factor of dilution requires more measurements of lithium abundance for stars located near and at the Hertzsprung gap, in particular for stars blueward of G0III, namely stars at the blue side of the gap. The distribution of lithium abundances before stars evolve along the giant branch may help us to understand the nature of the apparent discontinuity in lithium content around G0III and to establish on a more solid basis the dilution factor of stars evolving along the giant branch.

In this work we present new measurements of lithium abundance for giant stars in the spectral range F5III to G5III, typically stars located near and along the Hertzsprung gap. By combining these data with precise rotational velocities we analyse the rotation-lithium connection and the nature of the discontinuities in the distribution of these two stellar parameters.

\section{Observations}

For this study we have selected 54 luminosity class III giants with spectral types ranging from F5III to G5III. All these stars 
have been previously observed with the CORAVEL spectrograph (Baranne et al. 1979) for rotational velocity measurements and binarity signatures (De Medeiros \& Mayor 1999).

\subsection{Rotational velocity}

For most of the stars of the present sample, rotational velocity measurements were taken from The Catalog of Rotational and Radial Velocities for Evolved Stars by De Medeiros \& Mayor (1999). In this work, the authors present precise rotational velocity, $v \sin i$, for evolved stars, obtained on the basis of observations acquired with the CORAVEL spectrometers (Baranne et al. 1979). For giant stars of luminosity class III in particular, the measured rotational velocities have an uncertainty of about $1.0 \mathrm{~km} \mathrm{~s}^{-1}$ for stars with $v \sin i$ lower than about $30.0 \mathrm{~km} \mathrm{~s}^{-1}$. For faster rotators, the estimations indicate an uncertainty of about $10 \%$ on the measurements of $v \sin i$. For a few stars of the sample, most of them presenting evidence of high rotation, the $v \sin i$ value was determined on the basis of the spectral synthesis carried out through this work.

\subsection{Spectroscopic observations}

The spectral region around the lithium line at $6707.81 \AA$ was observed with two different telescopes. For northern stars, high-resolution spectra of the lithium region were acquired with the AURELIE spectrograph (Gillet et al. 1994) mounted at the $1.52 \mathrm{~m}$ telescope of the Observatoire de Haute Provence (France). The spectrograph used a cooled 2048-photodiode detector forming a $13 \mu \mathrm{m}$ pixel linear array. A grating with 1800 lines/mm was used, giving a mean dispersion of $4.7 \AA / \mathrm{mm}$ and a resolving power around 45000 (at $6707 \AA$ ). For this instrumentation and the selected set-up, the spectral coverage was about $120 \AA$. The signal to noise ratio was always better than 50. For southern stars, high-resolution spectra were acquired with the Coudé Echelle Spectrometer (CES) in the long camera mode (Kapper \& Pasquini 1996), mounted at the $1.44 \mathrm{~m}$ CAT telescope, at La Silla, ESO. A RCA high resolution $\mathrm{CCD}$ with $640 \times 1024$ pixels was used as detector, with a pixel size of $15 \times 15 \mu \mathrm{m}$. The dispersion was around $1.9 \AA \mathrm{Am}$ and the resolving power was about 95000 (at $6707 \AA$ ). The spectral coverage for this instrument was about $70 \AA$ and the signal to noise ratio was always better than 80 . For both observing runs, thorium lamps were observed before and after each stellar observation for wavelength calibration, whereas 3 series of flat-field using an internal lamp of tungstene were obtained during each night of observation. Flat-field corrections and wavelength calibrations were performed using the MIDAS package.

\section{Lithium abundances and spectrum synthesis}

In the present study, we adopted the spectral analysis method used by Lèbre et al. (1999) and Jasniewicz et al. (1999), in order to derive the lithium abundances from the resonance LiI line $(6707.81 \AA)$. We refer to these authors for a description of our abundance analysis assuming LTE. Nevertheless a few important points are described below.
To compute the more appropriated synthetic spectra to fit the observations, a first guess for the stellar parameters of our list of giants has been estimated. Thus we have looked into the literature and found effective temperatures $\left(T_{\text {eff }}\right)$, gravities $(\log g)$ and often metallicites ([Fe/H]) for 37 stars (over 54), mainly in Cayrel de Strobel et al. (2001), in Allende Prieto \& Lambert (1999), and in Alonso et al. (1999). For the rest of our sample (17 stars without any published stellar parameters determination), we first estimated $T_{\text {eff }}$ from $(B-V)$ colour index (Flower 1996), and we have set $\log g=3.0$, a microtuburlence velocity of $2 \mathrm{~km} \mathrm{~s}^{-1}$ and $[\mathrm{Fe} / \mathrm{H}]=0.0$ which are values commonly adopted for Pop. I giant stars.

Synthetic spectra were then computed from these stellar parameters and from the same line list and model atmospheres used in Lèbre et al. (1999) and Jasniewicz et al. (1999). The stellar parameters of all the giants (mainly $T_{\text {eff }},[\mathrm{Fe} / \mathrm{H}]$ and $v \sin i$ ) were then corrected, when necessary, in order to improve the fit quality of the several Fe I and other metallic lines found in the observed spectral range. The final adopted stellar parameters and the derived $\mathrm{Li}$ abundances are presented in Table 1 for all the stars of the sample, except HD 156015 which was too cool to accurately derive its effective temperature with the adopted procedure (however, the absence of Li signature is clear for this star). As already discussed by Lèbre et al. (1999), the major source of uncertainty for this abundance analysis is due to errors in the determination of the $T_{\text {eff }}$. We estimated that effective temperature were derived with an uncertainty smaller than $\pm 200 \mathrm{~K}$. This leads to an error of less than 0.2 dex on the derived metallicities and lithium abundances. We also checked with the work of Carlsson et al. (1994) that non-LTE effects can be neglected for the studied stars since these effects are always much smaller than 0.1 dex.

\section{Results and discussion}

\subsection{Rotation and $A_{L i}$ in the Hertzsprung gap}

The first step for the present analysis was the construction of the HR diagram to locate the evolutionary stage of the stars composing the working sample. In fact, such a procedure is important because in previous studies the only criterion for giant star classification was the spectral type. For this purpose, we have used HIPPARCOS (ESA 1997) trigonometric parallax measurements and $V$ magnitudes to compute absolute magnitudes and luminosities. Bolometric corrections $B C$ were determined from Flower (1996). Evolutionary tracks were computed from the Toulouse-Geneva code for stellar masses between 1 and $4 M_{\odot}$ and solar metallicity (see also do Nascimento et al. 2000 for complementary informations). The HR diagrams with the referred evolutionary tracks are displayed in Figs. 1 and 2. In addition, these figures show the behaviors of the lithium abundance and of the rotational velocity $v \sin i$, respectively. In these diagrams the dashed line indicates the evolutionary region where the subgiant branch starts, corresponding to the hydrogen exhaustion in stellar central regions, whereas the dotted line represents the beginning of the ascent along the red giant branch. Except for a few stars located along the subgiant branch, the large majority of stars in the present sample are 


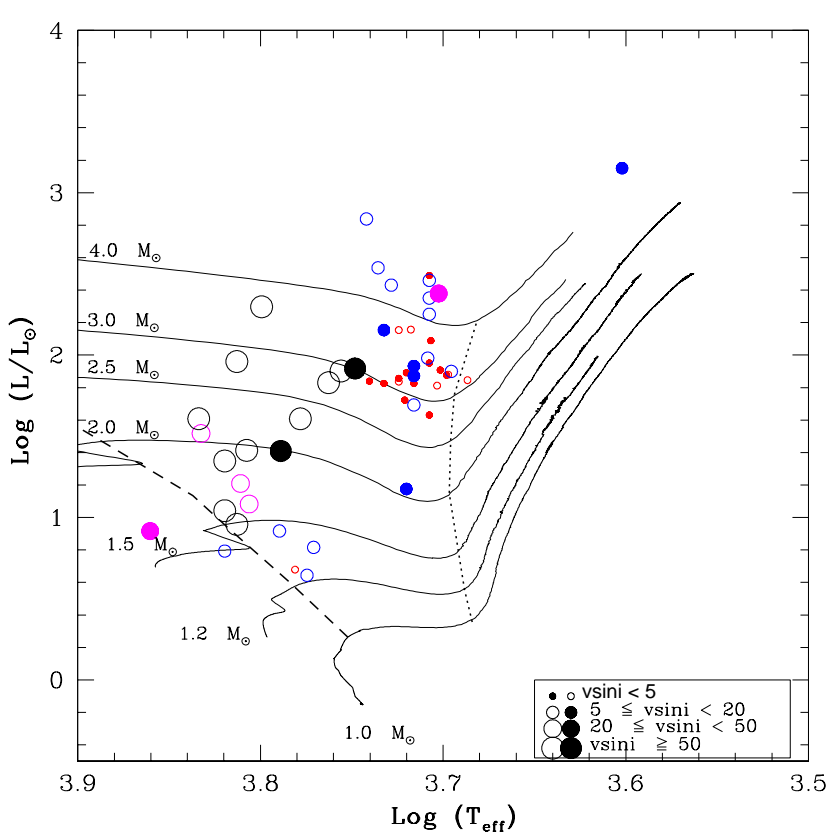

Fig. 1. Distribution of the rotational velocity of the stars in the HR diagram. Single and binary stars are identified with open and filled circles respectively. The size of the circles is proportional to the $v \sin i$ (in $\mathrm{km} \mathrm{s}^{-1}$ ). Luminosities have been derived from the Hipparcos parallaxes. Evolutionary tracks at $[\mathrm{Fe} / \mathrm{H}]=0$ are shown for stellar masses between 1 and $4 M_{\odot}$. The turnoff and the beginning of the ascent on the red giant branch are indicated by the dashed and dotted lines respectively.

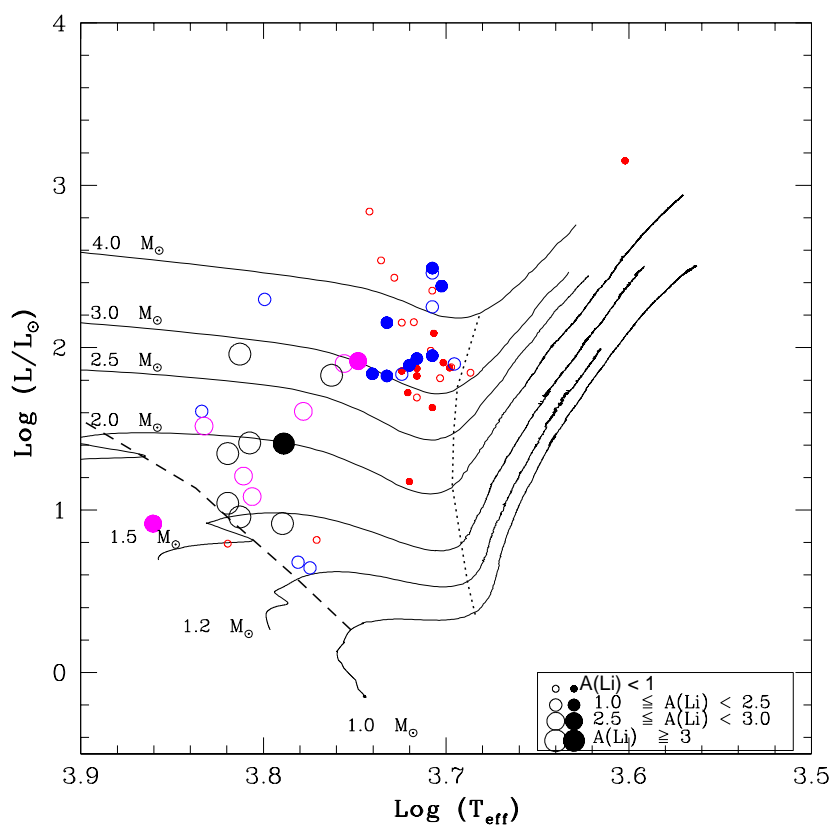

Fig. 2. Distribution of $\mathrm{Li}$ abundances in the HR diagram. Same as Fig. 1 except that the symbol size is proportional to the $\mathrm{Li}$ abundances.

effectively giants evolving prior to the ascent of the red giant branch.

Figure 1 shows the well established rotational discontinuity for giants of luminosity class III (e.g. De Medeiros \& Mayor 1990). As already shown by these authors, giants blueward of the spectral G0III (corresponding to the location of the rotational discontinuity, around $\log \left(T_{\text {eff }}\right) \approx 3.75$ ), show a wide range of rotational velocity from a few $\mathrm{km} \mathrm{s}^{-1}$ to about one hundred times the solar rotation. Giants redward of such spectral type (G0III) are essentially slow rotators, except for the synchronized binary systems and a dozen of late- $\mathrm{G}$ and $\mathrm{K}$ single giants for which the origin of their high rotation is not yet fully understood. At least for stars with turnoff mass greater than $1.5 M_{\odot}$, the sudden decline in rotation appears simply to reflect the rapid increase of the moment of inertia as the star evolves across this region of the HR diagram.

Figure 2 presents the behavior of lithium abundance, with a sudden decline in $A_{\mathrm{Li}}$ near the same spectral region where the discontinuity in rotation is observed. Nevertheless, it is quite clear from Figs. 1 and 2 that the behavior of rotation and lithium content in the Hertzsprung gap, as well as the location of both discontinuities, strongly depends on stellar mass. Such a fact shows that, for giant stars, it is not correct to define the location of the discontinuities in rotation and in $A_{\mathrm{Li}}$ at the same spectral type, as suggested by previous works based only on the analysis of the distributions of $A_{\mathrm{Li}}$ and $v \sin i$ versus spectral type or $(B-V)$ color index. Single stars located on the blue side of the Hertzsprung gap show a large spread in the lithium abundance, with values of $A_{\mathrm{Li}}$ ranging from less than 0.5 to $3.0 \mathrm{dex}$. In contrast, stars located at the red side show essentially low values of $A_{\mathrm{Li}}$, reflecting the dilution effects along this spectral region.

Clearly, one observes from Fig. 2 that stars with 2 to $3 M_{\odot}$ located redward of G0III $\left(\log \left(T_{\text {eff }}\right) \approx 3.75\right)$ show a factor of dilution of at least 600 , which is far in excess from the theoretical predictions. Such a fact points to an extra-mixing mechanism occuring before the beginning of the ascent of the red giant branch. The less massive giants show a similar disagreement between predicted and observed abundances. Brown et al. (1989) have measured lithium abundances for a large sample of late-G to $\mathrm{K}$ giants, with mean mass around $1.5 M_{\odot}$. For such stars, they found the mean lithium abundance lower than 0.0 dex, that is at least $\sim 1.5$ dex below the value predicted by standard theory. In Fig. 2 of the present paper, by taking into consideration the abundance of lithium for stars with masses between $1.5 M_{\odot}$ and $3.0 M_{\odot}$, located at the blue side of the Hertzsprung gap, and referring to a mean value of $A_{\mathrm{Li}}$ lower than 0.0 dex as estimated by Brown et al. (1989), we observe that the factor of dilution for this interval of mass is even more important than that observed for stars with $1.5 \mathrm{M}_{\odot}$.

It is interesting to point out that the binary systems seem to follow the same tendency presented by single stars. Redward of the spectral type G0III, the binary systems show essentially low values of $A_{\mathrm{Li}}$, reflecting also the effects of dilution along the Hertzsprung gap.

\subsection{The connection lithium - rotation in the Hertzsprung gap}

The dependence of lithium content on rotation along the Hertzsprung gap has been already reported in different works (Wallerstein et al. 1994; Alschuler 1975). For the present sample of stars, this trend is also clearly observed from Fig. 3, 


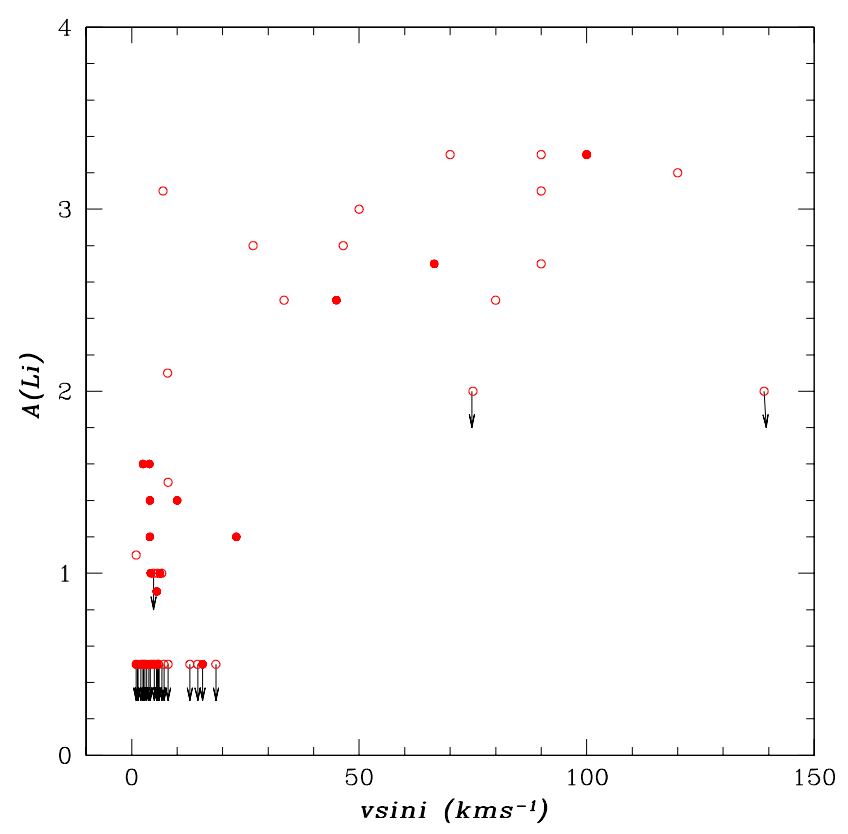

Fig. 3. Lithium abundance as a function of rotational velocity for giant stars in the Hertzsprung gap. Single and binary stars are identified by open and filled circles respectively. Vertical arrows indicate $A_{\mathrm{Li}}$ upper limits.

which displays lithium abundance as a function of rotational velocity. Stars with high rotation also present high values of $A_{\mathrm{Li}}$. In addition, the present data show a trend also observed for other luminosity classes, as well as for other spectral regions of giants. For slow rotators - in the present situation, for stars with $v \sin i$ lower than about $20 \mathrm{~km} \mathrm{~s}^{-1}$ - there is a large spread of the values of $A_{\mathrm{Li}}$, with a dispersion of at least 3 mag. Such a feature indicates that, also in the Hertzsprung gap, rotation is not the unique parameter driving $\mathrm{Li}$ abundance. Let us recall that, in spite of the limited number of binary systems with high $v \sin i$ value, the dependence of lithium on rotation seems to follow the same trend observed for the single stars. We have also analyzed the relation metallicity versus $v \sin i$ effects, looking for possible effects of metallicity on the spread observed in the $A_{\mathrm{Li}}$ versus rotation relation, by using data presented in Table 1. No clear trend arises from such an analysis.

\subsection{The connection lithium - deepening of the convective envelope}

Because the level of dilution of lithium depends on the level of convection in the stars, we analyse here the behavior of lithium abundance as a function of the deepening of the convective zone for our working sample. Such an analysis sounds interesting because most of the stars are crossing the Hertzsprung gap, where the convective zone is predicted to reach its most important development. For our purposes, we have first estimated the mass of each star $M_{*}$ from the HR diagram constructed for Figs. 1 and 2. Then, we have estimated the deepening in mass of the convective zone $M_{\mathrm{CZ}}$, according to the recipe applied by do Nascimento et al. (2000) and do Nascimento et al. (2003). In short, with the mass of each star in hand, we

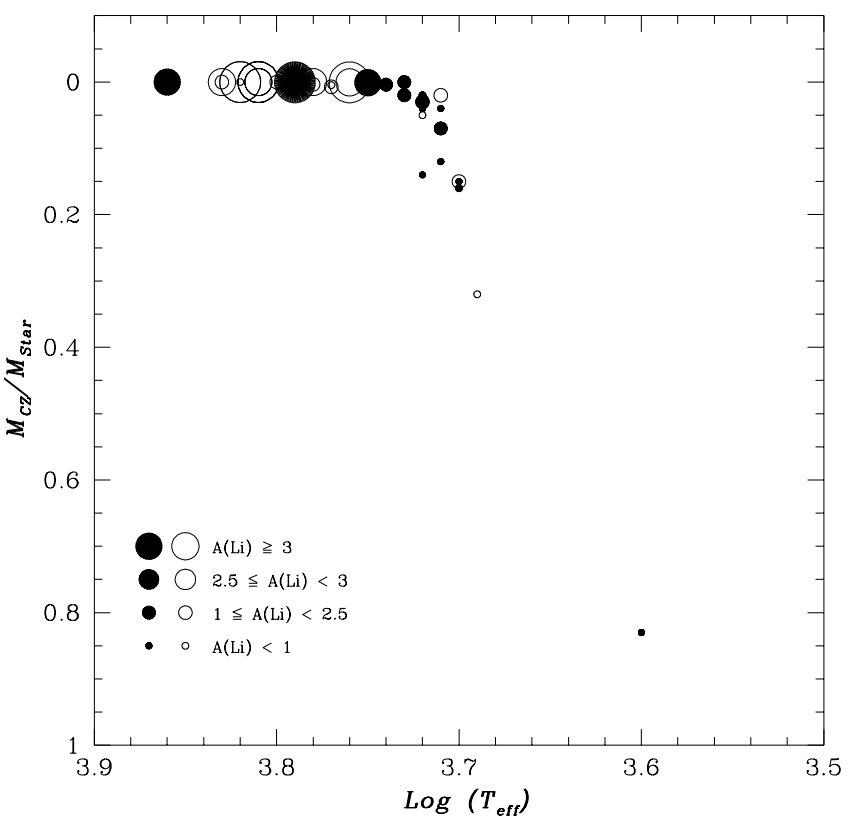

Fig. 4. The deepening (in mass) of the convective envelope as a function of the effective temperature for the stars in the present sample. Single and binary stars are represented by open and filled circles respectively. The symbol size is proportional to the $\mathrm{Li}$ abundances quoted in the figure.

have placed such mass in the convective zone mass deepening $M_{\mathrm{cz}} / M_{*}$ versus $T_{\mathrm{eff}}$ diagram from do Nascimento et al. (2000) and estimated the parameter $M_{\mathrm{cz}} / M_{*}$ for each star. The individual values of $M_{*}$ and $M_{\mathrm{cz}} / M_{*}$ are listed in Table 1 . The star HD 224342 is outside this analysis because of its very large and uncertain luminosity and mass. Figure 4 displays the deepening (in mass) of the convective envelope versus effective temperature, for single and binary stars. In this figure, the presence of the binary stars should be analysed with caution due to the large uncertainties in the estimation of mass for stars in binary sytems using evolutionary tracks. However it is clear from this figure that the sudden decline in $A_{\mathrm{Li}}$ in the Hertzsprung gap is directly associated with the rapid increase of the convective envelope in such region. Most of the stars with high Li content present an undeveloped convective zone, whereas stars with low Li content show a developed convective zone.

\section{Conclusions}

The present study brings precise abundances of Li for giant stars of luminosity class III evolving across the Hertzsprung gap. By combining these data with rotational velocity and other stellar parameters such as luminosity and effective temperature, we show a trend for the existence of a sudden decline in $A_{\mathrm{Li}}$ located around $\log \left(T_{\text {eff }}\right) \approx 3.75$, and corresponding to the spectral type G0III, where the well established rotational discontinuity for giants is defined. Blueward of this region, most of the stars with mass greater than about $1.5 M_{\odot}$ present a trend for high Li content, whereas for stars redward of this region $\mathrm{Li}$ is essentially diluted. The scenario of Li content in single stars seems to be followed by binaries. The dependence of Li content on rotation, in the sense that fast rotators show high $A_{\mathrm{Li}}$, 
Table 1. The stars of the present working sample with their physical parameters.

\begin{tabular}{|c|c|c|c|c|c|c|c|c|c|}
\hline HD & $\overline{(B-V)}$ & $T_{\text {eff }}$ & $\log g$ & {$[\mathrm{Fe} / \mathrm{H}]$} & $v \sin i$ & $M / M_{\odot}$ & $M_{\mathrm{cz}} / M_{*}$ & $A_{\mathrm{Li}}$ & Rem \\
\hline 87 & 0.901 & 5030 & 3.0 & -0.3 & 3.8 & 3.3 & 0.27 & $<0.5$ & SB? \\
\hline 895 & 0.68 & 5100 & 3.0 & -0.3 & 2.5 & 2.8 & 0.12 & $<0.5$ & SB \\
\hline 1671 & 0.442 & 6470 & 3.8 & -0.1 & 46.5 & 1.8 & 0.00 & 2.8 & \\
\hline 6903 & 0.697 & 5700 & 3.0 & -0.2 & 90.0 & 2.9 & 0.00 & 2.7 & (1) \\
\hline 17878 & 0.758 & 5300 & 2.7 & 0.1 & 2.6 & 3.8 & 0.02 & $<0.5$ & \\
\hline 48737 & 0.443 & 6600 & 3.9 & 0.0 & 70.0 & 1.6 & 0.00 & 3.3 & (1) \\
\hline 55052 & 0.397 & 6820 & 3.5 & 0.1 & 75. & 2.2 & 0.00 & $<2.0$ & \\
\hline 63208 & 0.571 & 5100 & 3.0 & 0.0 & 5.7 & 4.0 & 0.02 & 1.0 & \\
\hline 65448 & 0.58 & 5250 & 3.0 & 0.0 & 2.5 & 3.3 & 0.02 & 1.6 & SB \\
\hline 71369 & 0.856 & 5220 & 2.6 & 0.0 & 4.3 & 3.8 & 0.01 & $<0.5$ & \\
\hline 72779 & 0.681 & 5790 & 3.0 & 0.0 & 90.0 & 2.7 & 0.00 & 3.3 & (1) \\
\hline 74485 & 0.935 & 4960 & 2.1 & -0.4 & 6.6 & 3.3 & 0.25 & 1.0 & \\
\hline 74874 & 0.685 & 5400 & 3.0 & 0.0 & 4.0 & 3.0 & 0.00 & 1.4 & SBO \\
\hline 78715 & 0.89 & 5050 & 3.0 & -1.0 & 2.0 & 3.2 & 0.25 & $<0.5$ & \\
\hline 81025 & 0.774 & 5200 & 3.0 & -1.0 & 5.0 & 2.9 & 0.02 & $<0.5$ & \\
\hline 82210 & 0.781 & 5250 & 3.4 & -0.3 & 5.5 & 2.0 & 0.26 & 0.9 & SBO \\
\hline 85945 & 0.895 & 5200 & 3.0 & 0.0 & 6.2 & 3.4 & 0.02 & 1.0 & SB \\
\hline 92787 & 0.324 & 7250 & 4.2 & -0.3 & 45.0 & 1.6 & 0.00 & 2.5 & $(1,2), \mathrm{SB}$ \\
\hline 101133 & 0.401 & 6800 & 3.4 & 0.0 & 33.5 & 2.1 & 0.00 & 2.5 & \\
\hline 107700 & 0.515 & 5500 & 3.1 & -0.2 & 3.9 & 3.0 & 0.00 & 1.6 & SBO \\
\hline 108722 & 0.445 & 6600 & 3.6 & 0.0 & 100.0 & 1.9 & 0.00 & 3.3 & $(1)$ \\
\hline 111812 & 0.681 & 5600 & 3.0 & -0.3 & 66.5 & 3.0 & 0.00 & 2.7 & SBO \\
\hline 119458 & 0.857 & 5100 & 3.0 & -0.2 & 4.0 & 3.5 & 0.05 & 1.2 & SBO \\
\hline 121107 & 0.845 & 5100 & 3.2 & 0.0 & 14.5 & 4.0 & 0.02 & $<0.5$ & \\
\hline 136202 & 0.54 & 6040 & 4.0 & 0.0 & 4.8 & 1.2 & 0.00 & $<1.0$ & \\
\hline 140438 & 0.873 & 5110 & 3.0 & 0.1 & 5.8 & 3.5 & 0.05 & $<0.5$ & \\
\hline 151627 & 0.877 & 5090 & 3.0 & 0.0 & 4.1 & 3.8 & 0.04 & $<0.5$ & SB \\
\hline 152863 & 0.921 & 4980 & 3.0 & 0.1 & 2.9 & 3.2 & 0.25 & $<0.5$ & \\
\hline 153751 & 0.897 & 5040 & 2.0 & -0.3 & 23. & 4.0 & 0.05 & 1.2 & SBO \\
\hline 155646 & 0.504 & 6160 & 3.9 & 0.0 & 6.9 & 1.5 & 0.00 & 3.1 & \\
\hline 156015 & 1.164 & $<4000$ & - & - & 15.6 & 4.0 & 0.83 & $<0.5$ & SBO \\
\hline 157358 & 0.724 & 5300 & 3.0 & 0.0 & 1.0 & 3.1 & 0.05 & 1.1 & \\
\hline 159026 & 0.51 & 6300 & 3.0 & 0.0 & 139.0 & 3.7 & 0.00 & $<2.0$ & \\
\hline 160365 & 0.567 & 6150 & 3.4 & 0.0 & 100.0 & 2.0 & 0.00 & 3.3 & (1), SB \\
\hline 161239 & 0.683 & 5900 & 3.8 & 0.4 & 5.9 & 1.4 & 0.00 & $<0.5$ & \\
\hline 169985 & 0.487 & 5400 & 3.2 & 0.1 & 10.0 & 3.8 & 0.00 & 1.4 & (1), SB \\
\hline 173920 & 0.839 & 5350 & 3.0 & 0.0 & 8.0 & 4.0 & 0.01 & $<0.5$ & \\
\hline 175492 & 0.782 & 5100 & 3.0 & 0.0 & 4.2 & 4.0 & 0.01 & 1.0 & SBO \\
\hline 182900 & 0.456 & 6400 & 3.9 & 0.0 & 26.7 & 1.6 & 0.00 & 2.8 & (2) \\
\hline 185758 & 0.777 & 5440 & 3.1 & -0.1 & 7.1 & 4.0 & 0.00 & $<0.5$ & \\
\hline 200039 & 0.946 & 4990 & 3.0 & -0.4 & 1.0 & 3.1 & 0.25 & $<0.5$ & SB? \\
\hline 200253 & 0.994 & 5100 & 3.0 & 0.2 & 8.0 & 3.7 & 0.04 & 1.5 & (1) \\
\hline 202447 & 0.549 & 5300 & 3.2 & -0.4 & 1.3 & 3.1 & 0.03 & $<0.5$ & (2), SB \\
\hline 203574 & 1.005 & 4860 & 3.0 & -0.4 & 1.0 & 3.0 & 0.35 & $<0.5$ & \\
\hline 203842 & 0.474 & 6420 & 3.0 & -0.3 & 90.0 & 2.0 & 0.00 & 3.1 & (1) \\
\hline 208110 & 0.786 & 5260 & 3.0 & -1.0 & 3.3 & 3.0 & 0.05 & $<0.5$ & SB \\
\hline 209149 & 0.463 & 6500 & 3.8 & 0.0 & 50.0 & 1.5 & 0.00 & 3.0 & (1) \\
\hline 210459 & 0.471 & 6500 & 3.0 & 0.0 & 120.0 & 2.8 & 0.00 & 3.2 & (1) \\
\hline 214558 & 0.771 & 5200 & 3.0 & -0.4 & 1.4 & 3.1 & 0.03 & $<0.5$ & SB \\
\hline 215648 & 0.502 & 5950 & 4.0 & -0.4 & 7.9 & 1.2 & 0.00 & 2.1 & \\
\hline 218658 & 0.802 & 5200 & 2.9 & -0.2 & 5.5 & 3.3 & 0.02 & $<0.5$ & SBO \\
\hline 220657 & 0.617 & 6000 & 3.0 & 0.0 & 80.0 & 2.4 & 0.00 & 2.5 & (1) \\
\hline 223346 & 0.461 & 6600 & 4.1 & 0.0 & 18.5 & 1.5 & 0.00 & $<0.5$ & \\
\hline 224342 & 0.712 & 5520 & 2.0 & -1.0 & 12.8 & 4.5 & - & $<0.5$ & \\
\hline
\end{tabular}

(1) $v \sin i$ determined from the spectral synthesis. (2) Uncertain $T_{\text {eff }}$ or suspected binary. 
is also confirmed by the present set of data. In addition, the large spread in $A_{\mathrm{Li}}$ for slow rotators observed for other luminosity classes and other spectral regions occurs also in the Hertzsprung gap. Metallicity seems to have no effect on such a spread. Finally, we have combined the $A_{\mathrm{Li}}$ values with theoretical predictions on the convective zone deepening to analyse the extent of the level of convection on the Li dilution. It is clear from this analysis that stars with high Li content have mostly undeveloped convective zones, whereas stars with developed convective zone present low Li content.

Acknowledgements. J.R.M. warmly acknowledges colleagues of the Côte d'Azur Observatory at Nice, where a large part of this work was prepared during his stay in France. This work has been supported by continuous grants from the CNPq Brazilian Agency and by funds from the french Programme National de Physique Stellaire (INSU/CNRS). J.D.N.Jr. acknowledges CNPq for the fellowship PROFIX 540461/01-6. We also gratefully acknowledge C. Ferrari for her wonderful software tiramisu.

\section{References}

Allende Prieto, C., \& Lambert, D. L. 1999, A\&A, 352, 555

Alonso, A., Arribas, S., \& Martinez-Roger, C. 1999, A\&AS, 140, 261 Alschuler, W. R. 1975, ApJ, 195, 649

Baranne, A., Mayor, M., \& Poncet, J. L. 1979, Vistas Astron., 23, 279 Bonsack, W. K. 1959, ApJ, 130, 843

Brown, J. A., Sneden, C., Lambert, D. L., \& Dutchover, E. 1989, ApJS, 71, 293
Carlsson, M., Rutten, R. J., Bruls, J. H. M. L., \& Shchukina, N. G. 1994, A\&A, 288, 360

Cayrel de Strobel, G., Soubiran, C., \& Ralite, N. 2001, A\&A, 373, 159

De Medeiros, J. R., \& Mayor, M. 1990, in Cool Stars, Stellar Systems and the Sun, ed. G. Wallerstein (San Francisco: ASP), 404

De Medeiros, J. R., \& Mayor, M. 1999, A\&AS, 139, 433

De Medeiros, J. R., do Nascimento, J. D. Jr., Sankarankutty, S., Costa, J. M., \& Maia, M. R. G. 2000, A\&A, 363, 239

do Nascimento, J. D. Jr., Charbonnel, C., Lèbre, A., de Laverny, P., \& De Medeiros, J. R. 2000, A\&A, 357, 931

do Nascimento, J. D. Jr., Melo, C. H. F., Canto Martins, B. L., \& De Medeiros, J. R. 2003, A\&A, 405, 723

ESA 1997, The Hipparcos and Tycho Catalogues, ESA SP-1200

Flower, P. J. 1996, ApJ, 469, 355

Gillet, D., Burnage, R., Kohler, D., et al. 1994, A\&AS, 108, 181

Iben, I. 1965a, ApJ, 142, 1447

Iben, I. 1965b, ApJ, 141, 993

Kaper, L., \& Pasquini, L. 1996, CAT + CES Operating Manual, ESO Report, 3P6CAT-MAN-0633-0001

Lèbre, A., de Laverny, P., De Medeiros, J. R., Charbonnel C., \& da Silva, L. 1999, A\&A, 345, 936

Jasniewicz, G., Parthasarathy, M., de Laverny, P., \& Thévenin, F. 1999, A\&A, 342, 831

Rutten, R. G. M. 1987, A\&A, 177, 131

Wallerstein, G. 1966, ApJ, 143, 823

Wallerstein, G., Bohm-Vitense, E., Vanture, A. D., \& Gonzalez, G. 1994, AJ, 107, 2211 